\title{
Je lui trouve du charme : une structure particulière de la prédication seconde
}

\author{
Marleen Van Peteghem \\ Universiteit Gent \\ Marleen.VanPeteghem@UGent.be
}

\section{Introduction}

Le français possède une structure bi-transitive assez particulière dans laquelle le pronom datif $l u i^{1}$ ne correspond pas à un argument du verbe, sans pour autant pouvoir être supprimé (cf. (1)). Ces deux particularités l'opposent à la fois à la structure illustrée dans (2), où le complément datif correspond à un argument du verbe, et à celle dans (3), où le complément datif n'appartient pas à la structure argumentale du verbe et est omissible :

a. Je *(lui) croyais plus de talent.

b. Je *(lui) trouve mauvaise mine. (Olsson 1984)

a. J'ai donné un livre au garçon.

b. J'ai dit la vérité à mon collègue.
a. Je (lui) ai ouvert la porte.
b. Je (lui) ai préparé un café.

Sur le plan sémantique aussi, le complément datif de (1) présente des différences par rapport aux compléments datifs de (2) et de (3), dans la mesure où il ne reçoit pas de rôle sémantique du verbe, alors que les compléments datifs de (2) et de (3) ont un rôle sémantique typique du datif, respectivement de destinataire (angl. « recipient ») (2) et de bénéficiaire (3).

La structure présentée en (1) a été étudiée par Ruwet (1982)², qui la considère comme une construction à part qu'il appelle « construction à datif épistémique » (CDE). Ruwet analyse le complément datif comme le sujet d'un prédicat second, exprimé par l'objet direct, et propose donc une analyse de type 'small clause', sans avoir recours à ce terme encore trop récent à l'époque. Cette même analyse a été adoptée par plusieurs auteurs ( $c f$. Tremblay 1992, Van Peteghem 2006, Roberge \& Troberg 2007), mais est critiquée par Kupferman (2000), selon lequel il est impossible de rendre compte en syntaxe de ces structures, qui dépendent selon lui de «propriétés aléatoires, non systématiques » des verbes en question ( $c f$. Kupferman $2000: 80)$.

L'objectif de cette étude est de fournir des éléments de réponse aux deux questions suivantes : (i) quel est le rapport entre la construction à datif épistémique et les autres structures datives, et (ii) quel est le rapport entre la construction à datif épistémique et les autres structures de la prédication seconde. Ces questions s'inscrivent dans le débat mené dans les «Grammaires de constructions » («Construction Grammar»), qui postulent que chaque construction a un sens central, qui peut donner lieu à une polysémie constructionnelle. Nous montrerons que la $\mathrm{CDE}$ a un sens propre, dans la mesure où elle exprime l'évidentialité, sens qui apparaît également avec d'autres propositions réduites, comme par exemple la structure à attribut de l'objet (cf. Tobback 2008) et les comptes rendus de perception ( $c f$. Vogeleer 2008). De notre démonstration il ressortira que ce sens est une extension du sens premier de transfert de la structure dative.

Dans ce qui suit, nous résumerons d'abord les caractéristiques de ce type de datif telles qu'elles ont été décrites dans Ruwet (1982) et également dans Olsson (1984). Puis nous examinerons avec quels verbes apparait cette structure et quelle est la relation de la $\mathrm{CDE}$ avec les autres structures datives dans lesquelles 
entrent ces verbes. Ensuite nous examinerons en quoi la $\mathrm{CDE}$ se rapproche d'autres structures de la prédication seconde, notamment celle à attribut de l'objet.

\section{Le datif épistémique et l'analyse 'small clause'}

Le sens central des structures datives bi-transitives a souvent été décrit étant celui d'un transfert d'un objet par un agent vers un destinataire. Ainsi, Goldberg $(1995: 38)$ propose la description suivante du sens central de la structure bi-transitive en anglais :

\section{A. Agent successfully causes recipient to receive patient. (cf. give, throw, bring, etc.)}

En anglais ce sens donne lieu à différents autres sens reliés, décrits par Goldberg (ibidem) de la façon suivante :

B. Conditions of satisfaction imply that agent causes recipient to receive patient. (guarantee, promise, etc.)

C. Agent causes recipient not to receive patient. (refuse, deny)

D. Agent acts to cause recipient to receive patient at some future point in time. (bake, get, etc.)

E. Agent intends to cause recipient to receive patient. (leave, allocate, etc.)

E. Agent enables recipient to receive patient. (permit, allow)

Or, aucune de ces caractérisations ne peut rendre compte de la structure illustrée dans (1), qui n'exprime pas un transfert mais une attitude propositionnelle envers une prédication, qui, contrairement au transfert, n'exprime pas un événement, et n'implique aucun changement du monde.

Comme noté ci-dessus, le complément datif de la CDE n'est pas un argument du verbe, mais il ne peut néanmoins pas être supprimé. Cette particularité peut être expliquée par l'analyse de type 'small clause' proposée par Ruwet, le caractère obligatoire du complément datif étant dû à son statut de sujet de la proposition réduite. Cette analyse a également d'autres avantages dans la mesure où elle explique pourquoi la $\mathrm{CDE}$ permet souvent une paraphrase à l'aide d'une complétive :
a. Je croyais qu'il avait plus de talent.
b. Je trouve qu'il a mauvaise mine.

En outre, comme le montre Ruwet, elle explique pourquoi la CDE est possible uniquement avec des COD dénotant des propriétés ( $c f$. (5), voir Ruwet 1982: 174) et pourquoi le complément datif peut lier le pronom les uns les autres, ce qui confirme qu'il fonctionne comme un sujet syntaxique (cf. (6), voir Ruwet 1982 : 179) :
a. Je trouve qu'il a une femme insupportable.
b. *Je lui trouve une femme insupportable.
a. Je leur crois du respect l'un pour l'autre.
b. Je leur prête de mauvaises intentions l'un envers l'autre.

L'analyse comme 'small clause' amène Ruwet à rapprocher la CDE d'une autre structure à proposition réduite, à savoir la construction à attribut de l'objet (dorénavant $\mathrm{CAO}$ ), avec laquelle la CDE partage un certain nombre de propriétés ( $c f$. Ruwet $1982: 174)$ :

La plupart des verbes admettant la CDE (croire, trouver, imaginer, voir, deviner, proclamer, prétendre, supposer, etc.) se rencontrent également dans la $\mathrm{CAO}$, qui permet elle aussi le plus souvent la paraphrase par une complétive :

a. Je le croyais polygame. 

a'. Je croyais qu'il était polygame.
b. Je trouve cette fille très charmante.
b'. Je trouve que cette fille est très charmante.

La relation sémantique entre le datif et l'objet direct dans la CDE est la même que celle entre l'objet direct et l'attribut de l'objet dans la CAO : la CDE n'admet que des objets directs dénotant une propriété ( $c f .(5))$;

Des verbes comme croire et trouver diffèrent quant à la possibilité d'interroger sur l'attribut de l'objet, seul trouver permettant l'interrogation en comment. Or, des contraintes analogues s'observent dans la $\mathrm{CDE}$, où seul trouver admet l'interrogation en qu'est-ce que? (cf. Ruwet 1982:198) :

a. Comment le trouves-tu ? - Très intelligent.

b. *Comment le crois-tu ? - Très intelligent.

a. Qu'est-ce que tu lui trouves?

b. *Qu'est-ce que tu lui crois?

L'analogie entre les deux structures ressort particulièrement bien des deux exemples suivants, qui constituent des paraphrases l'une de l'autre :
a. Je lui trouve du charme.
b. Je le trouve charmant.

Comme l'ont montré Tremblay (1992), Herschensohn (1996) et Van Peteghem (2006), l'alternance entre accusatif et datif dans (10)a et (10)b dépend du cas assigné au prédicat second. Lorsque celui-ci n'a pas de cas, le sujet de la prédication seconde se trouve à l'accusatif; par contre, lorsque le prédicat second se trouve à l'accusatif, le sujet de la 'small clause' reçoit le cas datif, considéré comme « cas par défaut » par Tremblay et comme cas structural par Herschensohn et Van Peteghem. Le cas du sujet de la prédication seconde dépend donc dans une certaine mesure de la catégorie lexicale du prédicat second : lorsque ce dernier est adjectival, comme dans la $\mathrm{CAO}$, il ne reçoit pas de cas si bien que l'accusatif est disponible pour le sujet de la prédication seconde, alors que le datif est exclu ( $c f$. (11)b). Par contre, dans la CDE, le prédicat est un SN qui reçoit le cas accusatif, si bien que ce cas ne peut plus être assigné au sujet. Ce dernier reçoit par conséquent le cas datif :

$$
\begin{array}{lcc}
\text { a. Je le trouve charmant. } & v s & * \text { Je lui trouve charmant. } \\
\text { b. Je lui trouve du charme. } & v s & * \text { Je le trouve du charme. }
\end{array}
$$

Néanmoins des exemples comme (12) montrent que cette explication n'est pas suffisante car, comme l'avait déjà observé Ruwet $(1982: 198)$ dans une note, la CAO admet elle aussi des prédicats seconds nominaux, qui ne bloquent pas pour autant l'assignation de l'accusatif au sujet de la prédication seconde :

$$
\begin{aligned}
& \text { a. Je le / *lui crois mon meilleur ami. } \\
& \text { b. Valérie, son amie, le trouve le plus bel homme du monde ! ... (www.lepost.fr/../ } 1381582_{-}{ }^{\text {a- }} \\
& \text { voici-craint-on-moins-les-socialistes-que-les-sarkozy.html) }
\end{aligned}
$$

Ceci montre que la catégorie du prédicat second ne permet pas à elle seule de prédire le cas du sujet, mais que ce cas dépend aussi du type de relation prédicative, la CAO impliquant un rapport 'être', et la CDE un rapport 'avoir', verbe qui, comme l'ont bien montré des auteurs tels que Benveniste (1966), Guéron (1986), Tremblay (1992), Riegel (1998), etc., fonctionne également comme un verbe attributif, selon Guéron et Tremblay même dans ses emplois purement possessifs ${ }^{3}$ :
a. Je le trouve charmant.
a'. Je trouve qu'il est charmant.
b. Je le croyais professeur.
b'. Je croyais qu'il était professeur. 
c. Je le croyais à Paris.

a. Je le crois mon meilleur ami.

b. Je lui trouve du charme. c'. Je croyais qu'il était à Paris.

a'. Je crois qu'il est / *a mon meilleur ami.

b'. Je trouve qu'il a du charme.

Cependant, l'analyse 'small clause' de la CDE pose quelques problèmes :

(i) Ainsi, comme le notent Ruwet (1982) et Kupferman (2000), la CDE apparaît également avec des verbes bi-transitifs 'datifs', au sens de Leclère (1978), tels que prêter, promettre, qui n'admettent pas de complétive :

a. On prête/attribue beaucoup de charme à Marie. (cf. Kupferman $2000: 80$ )

b. *On prête / attribue que Marie a beaucoup de charme.

(ii) Les verbes de jugement n'admettent pas tous cette structure, ce qui fait conclure à Kupferman (2000: 80), qu' « on ne saurait y voir une dérivation syntaxique, générale » et que seul le lexique peut rendre compte de ces constructions :
a. *Paul jugeait du charme à Marie.
b. * Lea croyait une certaine impatience à Sam.
c. *Max savait du génie à cette œuvre-là.

\section{Le datif épistémique et les autres structures datives}

Examinons donc de plus près avec quels verbes apparaît la CDE et comment se situe la CDE par rapport aux autres structures datives dans lesquelles entrent ces verbes. Comme l'ont montré Ruwet (1982) et Olsson (1984), les verbes à CDE expriment le plus souvent la connaissance ou la perception, raison pour laquelle Ruwet a recours au terme d' "épistémique ». Ruwet et Olsson proposent tous deux une liste de ces verbes, qui a servi de point de départ de notre étude. Celle de Ruwet est une liste ouverte, dont la constitution n'est pas commentée ; celle d'Olsson se base sur un corpus fait avant l'ère informatique, à base de lectures de romans, et présente les verbes par ordre de fréquence dans cette structure (le chiffre entre parenthèses indique le nombre d'exemples trouvés par Olsson) :

(17) Liste de Ruwet (1982 : 172) : accorder, assurer, concevoir, contester, croire, découvrir, décréter, deviner, imaginer, inventer, postuler, prédire, pressentir, prétendre, présumer, prévoir, proclamer, promettre, reconnaître, refuser, souhaiter, soupçonner, subodorer, supposer, trouver, voir, vouloir, etc.

(18) Liste d'Olsson (1984 : 192) : (re)trouver (177), (res)sentir (156), (re)voir (98), connaître (93), découvrir (55), reconnaître (40), entendre (4), imaginer (6), supposer (8), croire (17), savoir (4), prêter (9), dénier (5), vouloir (3), deviner (1), remarquer (1), soupçonner (1), consentir (1), refuser (1), espérer (1), conserver (1), garder (1), tolérer (1), chercher (1).

A partir de ces deux listes, nous avons constitué une liste de verbes, dont nous avons examiné la fréquence dans un corpus d'exemples fait à partir de Frantext (tous genres, textes à partir de 1950). Nous avons retenu en première instance toutes les cooccurrences de ces verbes avec les pronoms lui/leur, même si cette requête exclut un grand nombre d'exemples :

(i) D'une part ceux contenant les datifs me, te, se, nous, vous, pronoms qui sont ambigus entre l'accusatif et le datif et dont le tri aurait demandé trop de temps. Ces cas seront inclus dans une étude ultérieure.

(ii) D'autre part notre requête a exclu également les exemples contenant un datif épistémique figurant sous forme de PP en [à $+\mathrm{SN}]$, comme dans (19), qui auraient également demandé un tri important étant donné l'ambiguité de la préposition $\grave{a}$, tri qui sera fait également pour une étude ultérieure : 


\section{Je trouvais un charme à cette vie retranchée. (Gracq - Frantext)}

Un autre problème, qui touche justement à la question qui nous intéresse ici, c'est que les structures datives trouvées avec certains de ces verbes ne pouvaient pas toutes être considérées comme des CDE. Certains verbes peuvent en effet prendre un complément datif d'un autre type, destinataire, bénéficiaire ou quelquefois même possessif. Nous avons donc classé les verbes d'après les types de structures datives autres que la $\mathrm{CDE}$ dans lesquelles ils peuvent entrer, ce qui nous a permis de distinguer 5 cas de figure, qui seront commentés brièvement dans ce qui suit :

1) des verbes datifs, à trois arguments, qui admettent un datif destinataire : accorder, attribuer, assurer, etc. ;

2) des verbes à deux arguments qui peuvent prendre un complément datif bénéficiaire: conserver, garder, chercher, trouver ;

3) des verbes de perception, qui entrent dans une structure dative lorsqu'ils régissent une infinitive : voir, entendre, sentir ;

4) le verbe voir entre en outre dans la structure dative de la possession inaliénable ;

5) des verbes qui n'admettent pas d'autre structure dative que la CDE : connaître, concevoir, croire, etc.

3.1. Pour les verbes du type de accorder (394) ${ }^{4}$, assurer (324), attribuer (266), consentir (21), contester (13), dénier (17), prédire (14), prêter (418), promettre (212), reconnaître (125), refuser (211), souhaiter (111), qui ont une structure argumentale à trois arguments, la $\mathrm{CDE}$ se présente comme une extension sémantique de leur structure de base à trois arguments. Ces verbes expriment normalement un transfert, mais le sens épistémique apparaît lorsque le COD est abstrait et peut être interprété comme un prédicat, comme dans (20)a. L'emploi du verbe est toutefois très proche de celui où il a un sens de transfert, comme dans $(20) \mathrm{b}$ :

(20) a. Le second, dans son principe le plus profond, vise à libérer tous les hommes en les asservissant tous, provisoirement. Il faut lui reconnaitre la grandeur de l'intention. (Camus Frantext)

b. ainsi ces sociétés par actions n'ont pas d'actionnaires, ou bien ceux-ci n'exercent pas les prérogatives que leur reconnaît le droit commercial, (Chenot - Frantext)

Le sens épistémique est particulièrement fréquent avec des verbes tels que attribuer, prêter et reconnaitre, mais apparaît même avec le verbe donner, comme le note également Ruwet (1982 : 177) :

(21) a. vous lui prêtez des sentiments de sollicitude qu'il ne m'a guère prodigués jusqu'à présent. (Marcel Aymé - Frantext)

vs $\quad$ b. Au besoin, on lui prêterait peut-être la chaise longue... (Fallet - Frantext)

(22) Elle est rudement mieux qu'avant, on lui donne dix ans de moins - là j'avais exagéré exprès. (Seguin - Frantext)

Le glissement du sens de transfert vers le sens épistémique provient donc ici entièrement du sens du COD et peut être décrit comme l'assignation d'une propriété par le sujet du verbe au sujet du datif, sans qu'il y ait toutefois transfert.

3.2. Selon Olsson la CDE apparaît également avec les verbes conserver (65) et garder (80) d'une part, et chercher (108) et trouver (345) d'autre part, qui admettent une autre structure dative du type de (3), dans laquelle le complément datif exprime le bénéficiaire du processus (cf. lui faire un café, lui préparer un gâteau) (cf. (23)b, (24)b). Les problèmes que posent ces deux couples de verbes sont un peu différents. Conserver et garder peuvent se combiner avec un OD exprimant une propriété du complément datif, mais n'en prennent pas pour autant un sens épistémique : ils expriment alors la permanence d'un état et semblent fonctionner plutôt comme des factitifs d'état : 
(23) a. Un excès de torsion enlève à un fil une grande partie de son élasticité alors qu'une trop faible torsion lui conserve une certaine élasticité, mais au détriment de sa résistance. (Thiébaut Frantext)

vs b. J'écrivis encore quelques lettres à Monsieur Racine pour lui montrer qu'on lui conservait sa pension, son logement au château et ses emplois, [...] (Chandernagor - Frantext)

(24) a. Certes, sa démarche était bien simple : elle comptait lui garder l'aspect d'une visite banale, d'où toute émotion est prudemment écartée. (Yourcenar - Frantext)

vs b. Révoltés par l'insolence de ce geste sans précédent, après avoir banni Chalumot ils lui gardaient toujours un chien de leur chienne. (Gibeau - Frantext)

Ici aussi, si prédication seconde il y a, elle résulte uniquement du sens du COD, ce qui est probablement la raison pour laquelle Ruwet n'a pas inclus ces verbes dans sa liste.

Pour les deux autres verbes la situation est plus complexe. Le verbe trouver peut avoir deux sens : «découvrir » ou «considérer ». Dans le premier sens il est le pendant de chercher et n'admet pas de complétive, dans le second il prend un sens d'attitude propositionnelle et il admet une complétive. Dans son sens premier de « découvrir », il entre dans la structure à datif bénéficiaire :

(25) Laissez-le dormir, lui a-t-elle dit, quand il se réveillera vous irez lui chercher un taxi. (Duras Frantext)

Or, c'est surtout dans son second sens qu'il entre dans la CDE et qu'il fournit même les exemples les plus prototypiques de cette structure, retenus d'ailleurs également par Ruwet :
a. Je lui trouve mauvaise mine. (Olsson 1984)
b. Je lui trouve beaucoup de charme. (Ruwet 1982 : 172)

b. Pour en revenir au livre de Mauriac, je lui trouve une noblesse que j'appellerais désespérée si ce mot n'était en contradiction avec la foi profonde de l'auteur. (Green - Frantext)

Néanmoins il n'est pas toujours évident de distinguer entre sens épistémique et sens premier de trouver dans la structure dative, car même lorsqu'il signifie « découvrir » et qu'il fonctionne comme pendant de chercher, trouver admet des COD dénotant une propriété du complément datif :

(27) Dans le grenier, on voit à nu, avec plaisir, la forte ossature des charpentes. On participe à la solide géométrie du charpentier. La cave, on lui trouvera sans doute des utilités. (Bachelard Frantext)

(28) Quand se produisent avec ampleur des phénomènes qui attirent l'attention, on a tendance à leur chercher des causes générales, cosmiques ou autres; (Maurain - Frantext)

Autrement dit, même pour un verbe épistémique typique tel que trouver, la limite entre emploi épistémique et non épistémique n'est pas toujours claire. Notons d'ailleurs que le TLF signale la CDE sous le premier sens de trouver, celui de "découvrir», et non pas sous le second sens, celui de « considérer ». Etant donné la fréquence avec laquelle trouver 2 s'utilise dans la CDE (et d'ailleurs aussi dans la CAO, cf. infra), trouver peut être considéré comme un des verbes les plus prototypiques de cette structure.

3.3. Le cas des verbes de perception entendre (8), sentir (3), et voir (121) est encore différent. Entendre et voir admettent un complément datif lorsqu'ils régissent une subordonnée infinitive, la syntaxe de ces verbes étant alors proche de la structure à union de faire factitif, telle qu'elle est décrite dans Kayne $(1975 / 1977)$ et par beaucoup d'autres auteurs. Le pronom datif correspond ici à un argument sémantique de l'infinitif, généralement le sujet ou quelquefois l'OI :

(29) a. Que j'aimerais lui entendre dire un jour : «Urbain, je suis heureuse d'avoir un grand frère ; $[\ldots] »($ Philhes - Frantext) 
b. dans l'état où sont les choses et dans le train que je leur vois prendre, je ne veux plus m'occuper de rien qui me rappelle hors de moi (Guéhenno - Frantext)

Cette fois-ci la distinction entre les deux emplois peut donc être faite sur une base purement formelle : la présence de l'infinitif ( $\rightarrow$ structure à union) ou d'un COD $(\rightarrow \mathrm{CDE})$. Néanmoins il faut signaler que même dans la $\mathrm{CDE}$ ces verbes gardent généralement leur sens de base de perception et ne prennent pas un sens épistémique :

a. « Tous les hommes de ma famille boivent ; avec eux, je ne peux pas faire autrement », dit-il au matin quand il lui voit les yeux rouges. (Yourcenar - Frantext)

b. Parfois, je sursautais de lui entendre une voix juvénile. (Chamoiseau - Frantext)

Comme le montrent les chiffres figurant ci-dessus, la structure est fréquente surtout avec voir. Néanmoins avec sentir elle apparaît un peu plus que ne le suggèrent les chiffres, notamment dans une structure réfléchie, même si le pronom se n'est pas morphologiquement marqué pour le datif, ce qui amène le total pour sentir à 86 occurrences ( $c f$. aussi Olsson $1984: 204-205)$ :

\section{Il se sent une grande tendresse pour les vieux. (Berger - Frantext)}

3.4. Avec le verbe voir, on trouve des cas relevant des structures de la possession inaliénable, bien décrites entre autres par Guéron $(1983,1985)$ et Vergnaud \& Zubizaretta (1992). Ces exemples se caractérisent par le fait que le COD contient un SN défini dénotant une partie du corps. Comme l'ont montré Guéron et Vergnaud \& Zubizaretta, cette structure se caractérise par le fait que l'article défini est dans une relation anaphorique liée, au sens générativiste du terme, avec le pronom datif. Le COD ne fonctionne pas comme un prédicat second ici, ce qui ressort du fait que la paraphrase par une structure attributive en avoir est impossible :

$$
\begin{aligned}
& \text { a. Les campeurs en redemandaient. Ils étaient maigres. On leur voyait les côtes. (Poirot-Delpech } \\
& \text { - Frantext) } \\
& \text { a'. Ils avaient ??des/*les côtes. } \\
& \text { b. Sur la photo du mariage, on lui voit les genoux. (Ernaux - Frantext) } \\
& \text { b'. Elle avait ??des / *les genoux. }
\end{aligned}
$$

Cette structure semble très proche de celle illustrée en (33):

(33) a. Mme Bordes a une chemise décolletée, elle est maigre et ridée, elle a eu six enfants. Ses manches de chemise sont relevées, on lui voit les coudes noueux et secs. (Duras - Frantext)

b. « Tous les hommes de ma famille boivent; avec eux, je ne peux pas faire autrement », dit-il au matin quand il lui voit les yeux rouges. (Yourcenar - Frantext)

Dans ces exemples toutefois, le COD est accompagné d'une prédication seconde du type décrit entre autres par Furukawa $(1987,1996)$ et Riegel (1998), dans lequel le COD du verbe avoir est accompagné d'un attribut de l'objet (cf. Sylvie a les yeux bleus). Ces structures permettent donc la paraphrase en avoir :
a. Elle avait les coudes noueux et secs.
b. Elle a les yeux rouges.

Ici les deux structures, la possessive et l'épistémique, semblent se rencontrer. Par contre, le cas suivant relève entièrement de la $\mathrm{CDE}$, dans la mesure où le trait définitoire de la structure de la possession inaliénable, à savoir la présence d'un article défini lié, n'est pas présent :

(35) Quand soudain Massignon se releva - [...] - je lui vis un visage extraordinaire : ravi, absent, brillant de larmes. (Nourissier - Frantext) 
3.5. Les autres verbes des listes de Ruwet et Olsson sont tous des verbes qui n'ont pas d'autre structure dative que la CDE. Pour certains de ces verbes nous n'avons trouvé dans Frantext aucune occurrence avec lui/leur à partir de 1950. C'est le cas de décréter, espérer, postuler, pressentir, présumer, prétendre, proclamer, remarquer, ressentir, retrouver, revoir, subodorer. En étendant la recherche à la période 1900-2009, nous avons tout de même trouvé quelques exemples pour les verbes décréter et remarquer:

(36) a. Il n'est presque aucun de ses livres où on ne le voie d'abord, s'emparant de termes, par exception, assez bien fixés, leur décréter des sens tout neufs : changeant, parfois, d'ouvrage à ouvrage ; volontairement éloignés, en tout cas, de la commune pratique. (Bloch - Frantext)

b. Depuis plusieurs jours, je lui remarquais des yeux cernés. (Genet - Frantext)

Il serait donc dangereux d'affirmer que ces verbes n'entrent pas dans la CDE, mais il est clair qu'ils sont rares dans cette structure.

Ce n'est donc que pour les verbes suivants que nous avons trouvé des exemples clairs de CDE ne pouvant pas être considérée comme une extension d'une autre structure dative :

concevoir (2), connaître (139), croire (16) $)^{5}$, découvrir (32), deviner (1), imaginer (4), inventer (24), prévoir (2), savoir (3), soupçonner (5), supposer (18), vouloir (148).

Les plus fréquents sont : connaître (139), croire (16), découvrir (32), inventer (24), sentir (86), supposer (18), trouver (246), voir (121), vouloir (148). Tous ces verbes ont en commun d'admettre un COD ; par contre, ils n'entrent pas tous dans une structure à complétive, notamment connaître, un des verbes les plus fréquents dans cette structure.

\section{Le datif épistémique et la prédication seconde}

Examinons maintenant les rapports entre la $\mathrm{CDE}$ et d'autres structures de la prédication seconde. En première instance je me pencherai sur la question de savoir si le COD fonctionne véritablement comme un prédicat dans cette structure, ce qui m'amènera à discuter la distinction entre avoir «attributif» vs «possessif» (cf. entre autres Benveniste 1966, Guéron 1986, Tremblay 1992, Tellier 1992, Kupferman 2000). Puis j'examinerai quels verbes à CDE admettent également la CAO et inversement, ce qui nous permettra d'analyser la relation entre ces deux structures de la prédication seconde.

\subsection{Le COD comme prédicat ou avoir comme verbe attributif}

Les COD qu'on trouve dans les CDE contiennent souvent un nom abstrait, dénotant une propriété, un état ou une action, qui conviennent bien comme prédicats sémantiques, ce qui ressort du fait que la CDE donne lieu à une paraphrase en [avoir $+\mathrm{SN}$ ], paraphrasable à son tour par [verbe attributif + adjectif] (être dans (39)a, avoir l'air dans (39)b) ou par un prédicat verbal (sourire dans (39)c) :

(39) a. La méfiance qu'elle devinait chez Francine lui donna-t-elle l'intuition que Paul l'aimait ? Elle lui connaissait à son égard de la bienveillance, et [...] (Monési - Frantext)

a'. Il avait de la bienveillance à son égard.

a"'. Il était bienveillant à son égard.

b. Elle lui trouvait l'air fatigué, « une drôle de figure » avait-elle commencé par dire. (RobbeGrillet - Frantext)

b'. Il a l'air fatigué.

c. Je lui vis, pour la première fois, dans le plissement des yeux, quelque chose comme un sourire. (Pontalis - Frantext)

c'. Il souriait. 
Néanmoins, le nom contenu dans le COD peut dénoter autre chose qu'une propriété, comme par exemple une partie du corps, un vêtement ou une relation de parenté. Dans la majorité des cas le COD contient un modifieur adjectival ou autre, qui apporte l'information prédicative centrale et la paraphrase en avoir est à son tour une structure de la prédication seconde, telle que décrite entre autres par Furukawa $(1987,1996)$, Riegel $(1988,1998)$. La paraphrase par une structure attributive en être est possible dans ce cas :

(40) a. «Tous les hommes de ma famille boivent; avec eux, je ne peux pas faire autrement», dit-il au matin quand il lui voit les yeux rouges. (Yourcenar - Frantext)

b. Il avait les yeux rouges.

c'. Ses yeux étaient rouges.

(41) a. On lui voit une bourse de cuir attachée aux reins. (Claudel - Frantext)

b. Il avait une bourse de cuir attaché aux reins.

c. Sa bourse de cuir était attachée à ses reins.

Par contre, dans d'autres cas, le COD ne comporte pas de modifieur et seule la paraphrase en avoir est possible, celle par [être + attribut] étant exclus. L'information nouvelle résulte alors surtout de la négation (42) ou d'un déterminant (43) :

(42) a. Meinthe [...] était devenu pour nous un personnage dangereux que nous sentions rôder la nuit autour de la villa. Nous ne lui connaissions pas de visage et de ce fait, il devenait de plus en plus obsédant. (Modiano - Frantext)

a'. Il n'avait pas de visage.

b. On lui connaît pas d'homme ! et qu'est-ce que ça prouve ? (Simonin - Frantext)

b'. Elle n'avait pas d'homme.

(43) a. Mon frère et moi nous lui connaissions au moins deux amants. (Lanzmann - Frantext)

a'. Il avait au moins deux amants.

b. On lui connaissait plus d'un amant dans la région; (Germain - Frantext)

b'. Il avait plus d'un amant dans la région.

Beaucoup d'études ont été consacrées à avoir comme verbe attributif, qui défendent deux points de vue différents :

- Benveniste (1966), Guéron (1986) et Tremblay (1992) plaident pour un traitement unitaire des constructions en avoir et considèrent avoir comme un verbe attributif au même titre que être ;

- Par contre, d'autres auteurs tels que Tellier (1992) et Kupferman (2000) jugent qu'il faut distinguer deux types de avoir: avoir possessif, qui permet la paraphrase par posséder (cf. (44) vs avoir attributif, qui met en jeu une prédication seconde (cf. (45)) :

a. Marie a un fils.

b. Posséder un fils comme celui-là n'est pas de tout repos pour les parents. (Kupferman $2000: 74)$

(45) a. Elle avait sa fille de malade.

b. Sa fille était malade.

Nous n'entrerons pas dans ce débat ici et nous nous limiterons à signaler que la CDE peut accueillir les deux types de relation-avoir. Notons toutefois que les emplois "possessifs », comme dans (42) et (43), résistent au critère de reconnaissance classique de la paraphrase en posséder, mais s'opposent néanmoins 
aux emplois attributifs dans la mesure où le COD ne comporte pas de prédicat second, contrairement à ce qui est le cas dans (40) et (41).

Or, notre hypothèse est que c'est cette valeur attributive de avoir en français qui rend la CDE possible, comme d'ailleurs aussi les autres types de structures datives. En effet, selon Barnes (1980) et Herslund (1988), la valeur de base de la structure dative en français est qu'elle exprime une relation « avoir ». Selon Herslund, la structure dative véhicule d'ailleurs toujours une prédication seconde mettant en jeu une relation avoir, même dans ses emplois purement attributifs, relation qu'il représente de la façon suivante : S causer (OI avoir O) ( $c f$. Herslund 1988 : 313). Pour vérifier cette hypothèse sur le rapport entre le sens de «avoir» et les types de datif, il serait intéressant d'examiner si la non-existence de la $\mathrm{CDE}$ dans des langues telles que l'anglais, le néerlandais, l'allemand, et aussi des langues romanes comme le roumain, est en rapport avec l'absence de sens attributif du verbe « avoir » dans ces langues.

\subsection{CDE vs CAO : les verbes}

Passons maintenant à la relation entre la $\mathrm{CDE}$ et la $\mathrm{CAO}$. Pour ce qui est des verbes entrant dans la $\mathrm{CDE}$, une confrontation de notre liste restreinte figurant sous 3.6. avec celles établies par Tobback (2005) ${ }^{6}$, en vue de sa thèse de doctorat sur l'attribut de l'objet, montre que tous ces verbes sont attestés également dans la CAO. Par contre, les listes de Tobback des verbes admettant la CAO contiennent beaucoup de verbes n'entrant pas dans la CDE, tels que les factitifs d'état (rendre, faire), les verbes de nomination (nommer, élire), mais également des verbes d'attitude propositionnelle, tels que considérer, estimer, penser, présumer, prétendre, qui sont proches de ceux admettant la CDE sans pour autant l'admettre?

En fait, tous nos verbes appartiennent à un sous-groupe de verbes d'attitude propositionnelle que Tobback (2008) appelle les « verbes de perception sensorielle ou cognitive », d'après González-García (2006). Ces verbes ont la particularité de se prêter facilement à des emplois parenthétiques ( $c f$. Schneider 2007) et à des emplois en tant que marqueurs discursifs ( $c f$. Dostie 2004). Selon ces auteurs, il s'agit de verbes qui tendent vers un emploi faible dans lequel ils ne participent pas au contenu propositionnel des énoncés. Ils servent alors de modalisateurs et ont surtout une fonction pragmatique.

Ceci amène Tobback (2008) à réexaminer le rôle de ces verbes dans la $\mathrm{CAO}^{8}$. Comme il ressort de son étude, plusieurs de ces verbes ont une préférence nette pour certaines structures syntaxiques dans lesquelles ils « ne se comportent pas tout à fait comme des verbes pleins» (Tobback 2008: 80) :

- le passif et surtout le participe passé passif, fréquent avec juger, dire, présumer, supposer :

(46) Depuis la mort du président hutu Juvénal Habyarimana, le 6 avril dernier dans un accident d'avion, présumé attentat, des combats opposent le Front patriotique rwandais. (Le Monde - Tobback 2008 : 81)

- la construction pronominale, qui apparaît surtout avec montrer, sentir, prétendre et vouloir :

En être réduit, dans une République qui se voulait celle du progrès social, à tenter de définir à quel prix il est possible de subsister sans perdre la vie, c'est, avouons-le, un bien pauvre progrès. (Le Monde - Tobback $2008: 83$ )

- le pronom sujet on, qu'on trouve surtout avec deviner, mais également avec connaître, croire, imaginer, penser et savoir :

(48) Cette actrice, qu'on a toujours connue nuancée, sobre, et comme éclairée de l'intérieur, est l'âme du film et porte son personnage à des dimensions «bressonniennes ». (Le Monde - Tobback $2008: 85$ )

Comme le montre Tobback, dans certains de ces cas ils servent alors de marqueurs évidentiels dans la mesure où ils permettent d'exprimer la source de l'information, mais le plus souvent ils expriment « l'attitude épistémique ou cognitive du locuteur sans référer à la source d'information » (Tobback 2008 : 81). Tobback note toutefois qu'il s'agit uniquement d'une tendance, qui ne se vérifie pas dans tous les cas et pas de la même façon pour tous les verbes. 
Or, on observe des phénomènes analogues pour les verbes dans la CDE. Certains de ces verbes apparaissent également surtout dans des structures pronominales, comme par exemple croire, sentir, découvrir (cf. aussi supra 3.3., exemple (31)) :

(49) a. As-tu jamais songé à ce qu'est ton public ? C'est celui qui, de tout temps, s'est trompé, qui achète les barbouillages à la mode, qui se croit du goût parce qu'il a de l'argent. (Dutour Frantext)

b. Mais pour la suite, Adrien se sentait un si grand courage dans les bras qu'il ne pouvait pas préciser jusqu'ou irait son projet d'ascension sur l'échelle sociale. (L'Hôte - Frantext)

c. Depuis la mémorable veste que ton grand-père a ramassée aux dernières élections, ils se sont complètement retirés, ils vivent en robe de chambre et se découvrent une maladie par jour. (Bazin - Frantext)

En outre, ces verbes ont tendance à apparaître dans des collocations (cf. se croire le droit de, se croire des droits - (ne pas) se sentir la force de - se sentir le cour ADJ) :

(50) a. je me fis une règle de n'accepter pour l'état ni moi-même aucun legs sur lequel des héritiers directs pourraient se croire des droits. (Yourcenar - Frantext)

b. Il n'avait plus besoin de cette colère qui l'avait fouaillé, il se sentait la force d'aller seul jusqu'au bout de sa route. (Clavel - Frantext)

c. La mère se sentait le cour serré. (Clavel - Frantext)

D'autres verbes apparaissent surtout dans des relatives, comme c'est le cas de connaître, qui figure dans une relative dans 100 des 139 exemples, c.-à-d. dans $71 \%$ des cas :

(51) a. Ma mère arriva le lendemain de bonne heure. Elle portait une de ses robes que je lui connaissais ; mais elle était coiffée d'un madras noir à petites rayures blanches. (Zobel Frantext)

b. -ah ! Laisse-moi tranquille avec cette histoire, dit Josette d'une voix irritée qu'Henri ne lui connaissait pas. (Beauvoir - Frantext)

Un autre constat qui va dans ce sens, c'est que beaucoup de ces verbes s'utilisent surtout dans des propositions négatives. C'est le cas surtout de connaître et de savoir. Ainsi pour le verbe connaitre, $47 \%$ de nos exemples présentent un contexte négatif (66/139, c.-à-d. 47\%) :

(52) a. - Ses hardes ne valaient pas trois liards. J'ai tout de suite prévenu sa fille. - Nous ne lui savions pas une fille », dit Nathanaël, incluant sans même le remarquer Monsieur Van Herzog dans sa réponse. (Yourcenar - Frantext)

b. On ne lui connaissait aucun ami à Lahore. (Duras - Frantext)

Dans ces contextes négatifs, le rôle du verbe est clairement modal. Il semble donc que, tout comme c'est le cas dans la CAO, le verbe de la CDE ne fonctionne pas comme le prédicat principal de la phrase au niveau sémantique, ni même toujours au niveau syntaxique, étant donné sa grande fréquence dans les relatives. Son rôle consiste surtout à exprimer soit la source de l'information, soit l'attitude du locuteur par rapport à la prédication seconde. Des analyses de corpus plus poussées devraient montrer pour chaque verbe quel est son emploi préférentiel. Ainsi connaître apparaît surtout avec des pronoms déictiques de $1^{\mathrm{e}}$ personne (surtout nous) et exprime alors l'attitude du locuteur, alors que croire apparaît surtout à la troisième personne et exprime donc surtout la source externe.

La valeur modalisatrice de la CDE est en rapport avec un autre effet sémantique souvent observé, à savoir l'absence de non-véridicité de la prédication seconde, signalée par Higginbotham (1984) comme caractéristique des verbes de perception dans la structure infinitive, qui est une autre structure de type 'small clause' : 
La plupart des verbes à $\mathrm{CDE}$ véhiculent en effet une présupposition de non-véridicité de la prédication seconde. C'est le cas de imaginer, inventer, soupçonner, supposer et vouloir.

En outre, comme l'avait déjà signalé Ruwet (1982), lorsque la CDE est en concurrence avec une complétive, la CDE s'oppose à la complétive par le fait qu'elle implique la non-véridicité de la prédication, alors que la complétive est neutre à cet égard. Ainsi la phrase (54)a laisse entendre que Pierre n'a pas vraiment des ennemis partout, effet sémantique qui n'est pas présent dans (54)b. Le même contraste sémantique s'observe dans la $\mathrm{CAO}$ et la complétive correspondante, effet sémantique qu'on observe également dans la CAO (cf. (55)) :

a. Pierre se croit des ennemis partout.

a. Pierre se croit malade.
vs
b. Pierre croit qu'il a des ennemis partout.
vs
b. Pierre croit qu'il est malade.

Notre hypothèse est donc que, du point de vue sémantique, la CDE est avant tout une structure évidentielle, servant à exprimer la source de l'information. Elle rejoint en cela la CAO de certains verbes, notamment les «verbes de perception sensorielle ou cognitive », mais la CAO a beaucoup d'autres valeurs sémantiques. Cette différence sémantique entre les deux structures explique pourquoi la CDE apparait avec beaucoup moins de verbes que la CAO.

\section{Conclusion}

L'analyse qui précède a fourni des éléments de réponse aux deux questions que nous nous sommes posées au début :

- Pour ce qui est de la relation de la CDE par rapport aux autres structures datives, nous avons pu constater que cette relation varie selon le verbe. Pour certains verbes la CDE est simplement une extension du sens de transfert vers celui d'assignation d'une propriété, sens très fréquent avec des verbes datifs tels que prêter, attribuer ou reconnaître. Probablement c'est cette extension qui est à la base de la $\mathrm{CDE}$ avec des verbes non datifs épistémiques. Une analyse diachronique devrait montrer si l'emploi épistémique de ces verbes non datifs est en effet ultérieur à celui des verbes du type attribuer.

- Quant à la relation de la CDE avec d'autres structures de la prédication seconde, on constate que les verbes non datifs entrant dans la $\mathrm{CDE}$ peuvent tous entrer également dans la $\mathrm{CAO}$, mais que l'inverse n'est pas vrai. Seuls des verbes d'attitude propositionnelle à emploi «faible», exprimant une perception sensorielle ou cognitive, le peuvent.

On peut donc conclure que la valeur sémantique de la CDE est d'exprimer l'assignation d'une propriété par le sujet propositionnel au référent du datif. Il s'agit là d'une extension sémantique du sens de base de transfert de la structure dative, extension est probablement facilitée en français par la valeur attributive du verbe avoir, verbe sous-jacent à la majorité des structures datives en français, comme l'ont montré Barnes (1980) et Herslund (1988). Ceci nous amène à l'hypothèse selon laquelle la CDE sert surtout à exprimer la source de la prédication et pourrait dans ce sens être décrit comme une structure de l'évidentiel. Il n'en reste pas moins que les verbes diffèrent beaucoup entre eux à cet égard. Pour vérifier cette hypothèse, une étude plus systématique d'un corpus plus important et plus complet (incluant les formes nécessitant un tri) est donc nécessaire. A suivre.

\section{Références bibliographiques}

Authier, J.-M. et Reed, L. (1991). Ergative Predicates and Dative Cliticization in French Causatives. Linguistic Inquiry, 22-1, 197-205.

Barnes, B. (1980). The Notion of 'Dative' in Linguistic Theory and the Grammar of French. Lingvisticae Investigationes, 6, 245-292.

Benveniste, E. (1966). Etre et avoir dans leurs fonctions linguistiques. Problèmes de linguistique générale 1. Paris : Gallimard. 
Dostie, G. (2004). Pragmaticalisation et marqueurs discursifs. Paris-Bruxelles : De Boeck/Duculot.

Furukawa, N. (1987). Sylvie a les yeux bleus : construction à double thème. Lingvisticae Investigationes, 11, 283-302.

Furukawa, N. (1996). Grammaire de la prédication seconde. Forme, sens et contraintes. Paris : Duculot.

Goldberg, A. E. (1995). Constructions. A Construction Grammar Approach to Argument Structure. Chicago/London : University of Chicago Press.

Gonzálvez-García, F. (2006). Passives without actives : evidence from verbless complement clauses in Spanish. Constructions, SV1-5/2006. (disponible via : www.constructions-online.de/articles/specvol1/679).

Guéron, J. (1983). L’emploi possessif de l'article français. Langue française, 58, 23-35.

Guéron, J. (1985). Inalienable Possession, Pro-inclusion and Lexical Chains. In Guéron, J., Obenauer, H.-G., Pollock, J.-Y. (eds). Grammatical Representation. Dordrecht : Foris, 43-86.

Guéron, J. (1986). Le verbe avoir. Recherches linguistiques de Vincennes, 14-15, 155-186.

Herschensohn, J. (1996). Case Suspension and Binary Complement Structure in French. Philadelphia / Amsterdam : John Benjamins.

Herslund, M. (1988). Le datif en français. Louvain / Paris : Peeters.

Higginbotham, J. (1984). Les comptes rendus de perception. Communications, 40, 1, 149-180.

Kayne, R. (1975 / 1977). Syntaxe du français. Le cycle transformationnel. Paris : Seuil. (Traduction de Kayne, R. 1975. French Syntax. Cambridge (Mass.) : MIT Press).

Kupferman, L. (2000). Avoir et la prédication seconde. Langue française, 127, 1, 67-85.

Leclère, C. (1976). Datifs syntaxiques et datifs éthiques. In Chevalier, J.-C. et Gross, M. (éds). Méthodes en grammaire française. Paris : Klincksieck, 73-96.

Leclère, C. (1978). Sur une classe de verbes datifs. Langue française, 39, 66-75.

Melis, L. (1996). The dative in Modern French. In Van Belle, W. et Van Langendonck, W. (eds). The dative and its counterparts. Vol 1 Descriptive studies. Amsterdam : Benjamins, 39-72.

Olsson, H. (1984). La construction je lui trouve mauvaise mine en français moderne. Studia Neophilologica, 56, 183213.

Riegel, M. (1988). L'adjectif attribut de l'objet du verbe avoir : amalgame et prédication complexe. Travaux de linguistique, 17, 69-87.

Riegel, M. (1998). De il a les yeux bleus à je n'ai pas le cinq-à-sept facile : les avatars de la construction attributive de avoir. Travaux et recherches en linguistique appliquée, série B, 1, 99-108.

Roberge, Y. et Troberg, M. (2007). Thematic indirect objects in French. Journal of French Language Studies, 17-3, 297-322.

Rooryck, J. (1988a). Critères formels pour le datif non lexical en français. Studia Neophilologica, 60, 97-107.

Rooryck, J. (1988b). Restrictions on dative cliticization in French causatives. Journal of Semantics, 6-1, 41-55.

Ruwet, N. (1982). Le datif épistémique en français et la condition d'opacité de Chomsky. In Ruwet, N. Grammaire des insultes et autres études. Paris : Seuil, 172-204.

Schneider, S. (2007). Reduced parenthetical Clauses as Mitigators. Amsterdam/Philadelphia : Benjamins.

Tellier, C. (1992). Remarques sur avoir attributif et possessif. Revue québécoise de linguistique, 22, 145-164.

Tobback, E. (2005). Les constructions à attribut de l'objet et le marquage de la relation prédicative seconde. Université de Gand : Thèse de doctorat.

Tobback, E. (2008). La structure à attribut de l'objet des verbes d'attitude propositionnelle : réflexions sur le rôle du verbe. Travaux de linguistique, 57, 73-88.

Tremblay, M. (1992). «Avoir ou être ». Revue québécoise de linguistique, 22, 1, 145-163.

Van Peteghem, M. (2006). Le datif en français : un cas structural. Journal of French Languages Studies, 16, 93-110. 
Vergnaud, J.-R. et Zubizaretta, M.-L. (1992). The Definite Determiner and the Inalienable Construction in French and English. Linguistic Inquiry, 23-4, 595-652.

Vogeleer, S. (1994). L'accès perceptuel à l'information : à propos des expressions un homme arrive / on voit arriver un homme. Langue française, 102, 69-83.

\footnotetext{
${ }^{1}$ Nous appelons «datifs» les clitiques lui/leur et les compléments en à que ces clitiques sont susceptibles de pronominaliser (Je parle à Pierre - Je lui parle), par opposition aux compléments en à non cliticisables (Je pense à Pierre - *Je lui pense), suivant en cela Kayne (1975/1977) et beaucoup d'autres auteurs parmi lesquels Leclère (1976, 1978), Barnes (1980), Herslund (1988), Rooryck (1988a, 1988b), Authier et Reed (1991), Melis (1996), etc.

${ }^{2}$ Elle a été étudiée également par Olsson (1984), qui, bien qu'il insiste également sur l'idée de prédicativité de l'OD, appelle ce type de datif « datif de localisation », ce qui suggère qu'il attribue le rôle de lieu au complément datif.

${ }^{3}$ Cette idée n'est pas partagée par tous les auteurs (cf. infra sous 4.1.). Pour des critiques, voir Kupferman (2000), qui considère qu'il faut distinguer entre deux verbes avoir : avoir 1 possessif et avoir 2 attributif.

${ }^{4}$ Les chiffres indiquent le nombre d'occurrences total du verbe en question avec les pronoms lui/leur, sans distinction entre datif lexical et datif épistémique.

${ }^{5}$ Pour croire, nous n'avons trouvé que 4 exemples avec lui/leur, ce qui est assez étonnant étant donné que tant dans l'étude de Ruwet que dans celle d'Olsson, croire apparaît comme un des verbes les plus typiques de cette structure. Nous avons alors étendu notre recherche aux emplois réfléchis, ce qui a amené le total d'exemples sur 16 cas. La même opération devra être faite pour les autres verbes de la liste, car, comme l'a montré Olsson, beaucoup de ces verbes apparaissent dans la CDE surtout dans la structure réfléchie.

${ }^{6}$ Nous remercions Els Tobback pour les listes qu'elle nous a mises à notre disposition.

${ }^{7}$ Sur Internet, nous avons trouvé les exemples suivants, qui ne sont toutefois pas acceptés par tous les locuteurs du français :
}

(i) Que celui qui prête à d'autres une opinion ou lui présume un comportement en dit plus sur lui-même que sur autrui. (dinersroom.eu/1605/vertueuse-jeunesse/)

(ii) Je préfère ne pas le penser, parce que si l'on pense qu'il y a une raison, ça présupposerait qu'on lui considère un positionnement précis. (www.agoravox.tv/article.php3?id article=24006)

(iii) [...] un des grands joueurs, je lui estime un grand parcours. (www.le10sport.com)

(iv) Je lui juge un beau naturel. (www.archive.org/.../analectesdubibl02gaygoog_djvu.txt)

(v) On lui prétend un pouvoir d'envoûtement exceptionnel, (alestyr_rpg.monforum.fr/les-angaivt694341.html?...)

\footnotetext{
${ }^{8}$ Les verbes examinés par Tobback sont : connaître, considérer, croire, découvrir, deviner, estimer, imaginer, juger,
} montrer, penser, présumer, savoir, sentir, supposer, trouver, voir, dire, prétendre, vouloir. 